\title{
Multidisciplinary Approach to the Conservative and Regenerative Management of Endo-Perio Lesion
}

\author{
R. Swaminathan ${ }^{1}$, J. Raghunathan ${ }^{1}$, Sivaram Subbiah ${ }^{2}$ \\ ${ }^{1}$ Department of Periodontics, Faculty of dental sciences, Sri Ramachandra University, Chennai, India \\ ${ }^{2}$ Department of Orthodontics, Penang International Dental College, Malaysia \\ Received July 26, 2014 Revised August 13, 2014; Accepted August 18, 2014
}

\begin{abstract}
This case report evaluates the clinical and radiographic response of biphasic calcium phosphate (ossify) when used in combination with bioresorbable membrane (sybograft - GBR) for the treatment of intrabony defect around multi-rooted teeth with an endodontic and periodontal lesion. A 47-year-old female patient with an endoperio lesion in the left mandibular first molar was initially treated with endodontic therapy. After endontic management of the teeth a full-thickness flap reflected, complete degranulation and root planing was performed. An allograft was then used to fill the defect and a GBR membrane was placed over the filled defect. Radiographs, clinical probing depths, and attachment levels were obtained before treatment, 3, 6, 9 and 24 months following treatment. Reduction in pocket depth and gain in clinical attachment level were observed during follow up. This case report demonstrates that proper diagnosis, followed by removal of etiological factors and utilizing the guided tissue regeneration technique combined with osseous grafting, will restore health and function of a tooth with severe bone loss caused by an endo-perio lesion. This report highlights a conservative and regenerative approach to the management of endo-perio lesion rather than a radical approach such as hemisection.
\end{abstract}

Keywords: Endo-perio lesion, Guided tissue regeneration, Bone Graft

Cite This Article: R. Swaminathan, J. Raghunathan, and Sivaram Subbiah, "Multidisciplinary Approach to the Conservative and Regenerative Management of Endo-Perio Lesion.” International Journal of Dental Sciences and Research, vol. 2, no. 4B (2014): 11-13. doi: 10.12691/ijdsr-2-4B-4.

\section{Introduction}

Clinicians often have difficulty in the diagnosis and treatment of the combined endodontal and periodontal (endo-perio) lesion. In some cases, the influence of pulpal pathology may create periodontal involvement whereas in others, periodontal pathology may create pulpal pathology. In the case of a combined endo-perio lesion, the endodontic therapy results in healing of the endodontic component of involvement while the prognosis of tooth would finally depend on the healing outcome of the periodontal structures. Periodontal surgical procedures have focused on the elimination or reduction of hard and soft tissue defects (i.e., probing depths and osseous defects) by regenerating new attachment. The pathways for the spread of bacteria between pulpal and periodontal tissues have been discussed with controversy. [1,2,3] Pulpal infection can drain through the periodontal ligament space and give an appearance of periodontal destruction, termed retrograde periodontitis. Similarly, both pulpal and periodontal infections can coexist in the same tooth, termed combined lesions, where the treatment depends on the degree of involvement of the tissues. Both endodontic and periodontal diseases are caused by a mixed anaerobic infection. The classification used for endodonticperiodontal lesions was given by Simon et al. (1972) [4]: 1- Primary endodontic lesions, 2- Primary endodontic lesions with secondary periodontal involvement, 3-
Primary periodontal lesions, 4- Primary periodontal lesions with secondary endodontic involvement, 5- True combined lesions.

In teeth with primary periodontal lesions with secondary endodontic involvement the apical progression of a periodontal pocket may continue until the apical tissues are involved. In this case, the pulp may become necrotic as a result of infection entering via lateral canals or the apical foramen. In single-rooted teeth, the prognosis is usually poor. In molar teeth, the prognosis may be better [5].

\section{Case Report}

A 47-year-old patient reported to the private practice with a complaint of food impaction in the lower left back tooth region for the past 1 year. On intraoral examination, none of the teeth had dental caries; a $10 \mathrm{~mm}$ periodontal pocket in the distobuccal aspect of 36 was found (Figure 1a). Further periodontal examination revealed no bleeding on probing, no obvious inflammatory changes in the gingiva, no gingival recession along the distal root, no mobility. An intraoral periapical radiograph revealed widening of periodontal ligament space and angular bone loss around the distal root of 36 extending upto the apex with radiolucency in the furcation region (Figure 2a). The tooth vitality was assessed with electric pulp testing which demonstrated a delayed response indicating it was partially vital. Based on the clinical and radiographic 
findings a provisional diagnosis of primary periodontal and secondary endodontic lesion in relation to 36 was made. Treatment planning was done taking into consideration that the tooth had a delayed response to pulp testing and the periodontal status of the tooth. Endodontic treatment was first done and the patient was followed up for 3 months. At the end of 3 months, IOPA was taken with 36 which showed that the furcation involvement and intrabony defect was still present (Figure 2b). On clinical examination, it was observed that there was no change in the soft tissue measurements. Therefore, a conservative treatment approach charted and regenerative periodontal surgery using of biphasic calcium phosphate and resorbable GBR membrane was planned for treatment of furcation and intrabony defect.
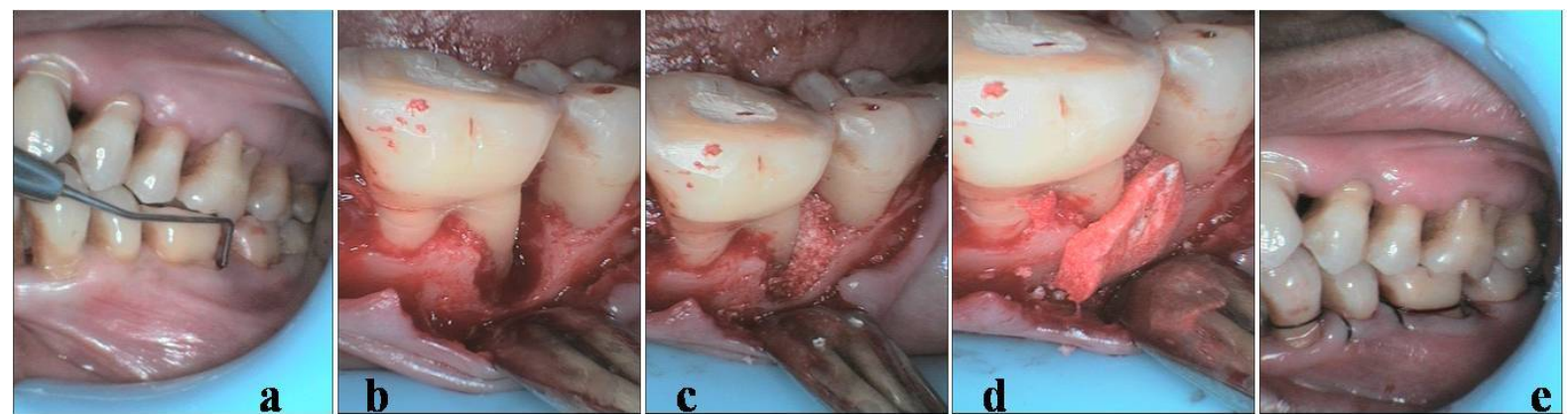

Figure 1. a- pre operative probing depth $10 \mathrm{~mm}$ in distobuccal aspect of 36; b- Full thickness mucoperiosteal flap elevated; c- Intrabony defect filled with one graft; d- GBR membrane placement; e- Flap sutured with 3-0 black silk continuous sling suture

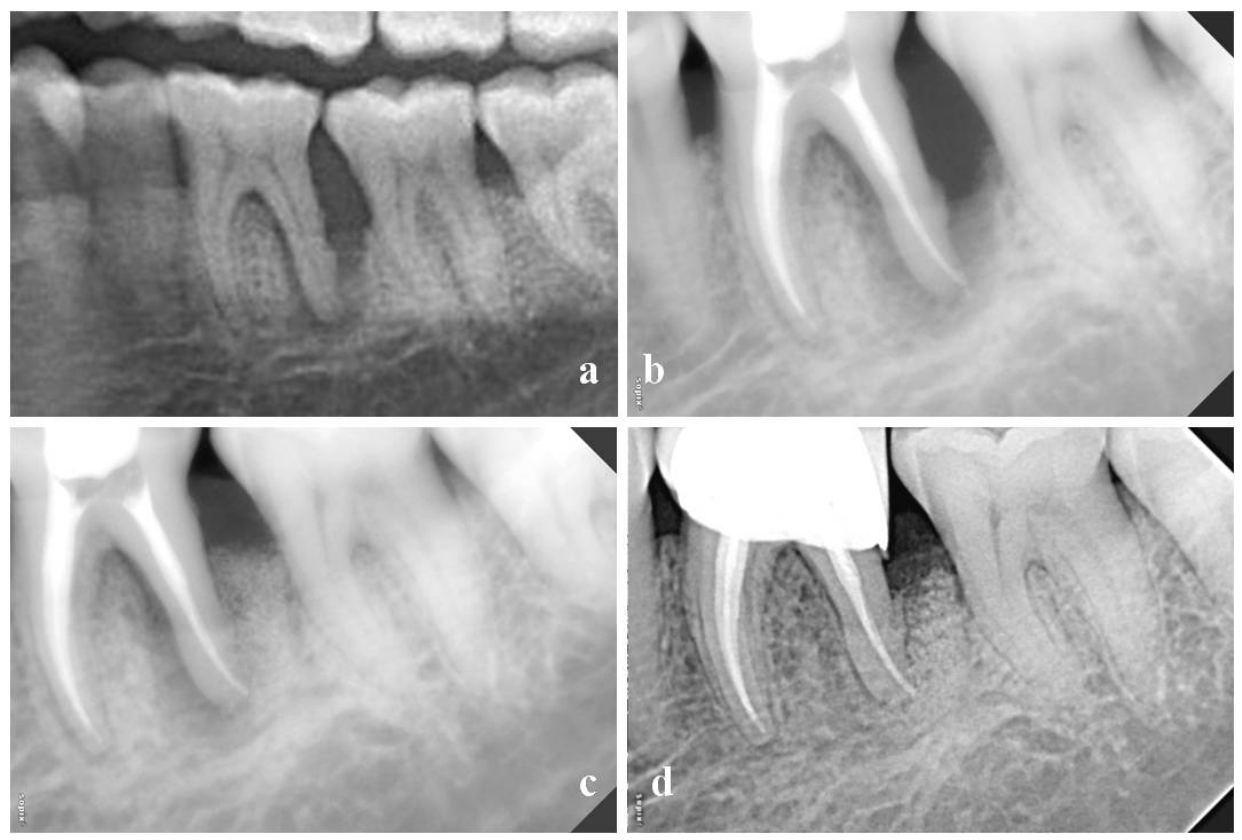

Figure 2. a- Pre operative IOPA 36; b- 3 months after endodontic management of 36; c- Immediate bone fill in 36 intrabony defect; d- 24 months post operative following placement of PFM in 36

\section{Surgical Procedure}

Under aseptic conditions and sterilization the periodontal surgery was performed as follows. Local anaesthesia was administered using xylocaine with adrenaline 1:200,000 in relation to 36. A full thickness mucoperiosteal flap was raised at the buccal aspect with intrasulcular incision (Figure 1b). No vertical incisions were given as good access could be achieved with horizontal incisions. Full thickness flap was raised till the base of intrabony defect. After complete reflection thorough debridement was done at the defect area using Gracey's curette \# 13 and 14. Scaling and root planning was carried out on the exposed root surface area of the defect. The intrasurgical assessment included morphology of the periodontal defect, defect type, material of choice to fill the defect and augment healing, control of patient's oral hygiene, and wound stabilization. [6,7] biphasic calcium phosphate material with osteoconductive properties was placed in the defect (Figure 1c). Bio resorbable GBR membrane was placed over the filled defect and stabilized (Figure 1d). Primary soft tissue closure of the flap was done with non-resorbable braided black silk (3-0) suture using continuous sling technique (Figure 1e). Coe-Pak was placed over the surgical area. Post operative instructions and medications (Cap.Amoxicyllin 500mg TDS, Tab. Paracetemol 500 mg TDS for 5 days) were given. The patient was prescribed $0.12 \%$ chlorhexidine mouthwash for rinsing twice daily. The sutures were removed 10 days after surgery. The patient was also advised to continue mouthwash for another 3 weeks.

The patient was put on regular recall at $1,3,6$, and 9 , 24 months. The bone graft resulted in a substantial bone fill (Figure 2c). After 6 months, the probing depth was found to reduce to $3 \mathrm{~mm}$ (Figure 3a). The post-op 
radiograph shows bone fill in the furcation defect as well as the intrabony defect. 3 months following periodontal surgery porcelain fused metal crown was placed in relation to 36 (Figure 3c). No bleeding on probing was found. The patient was periodontally maintained at 3month intervals throughout the entire treatment.
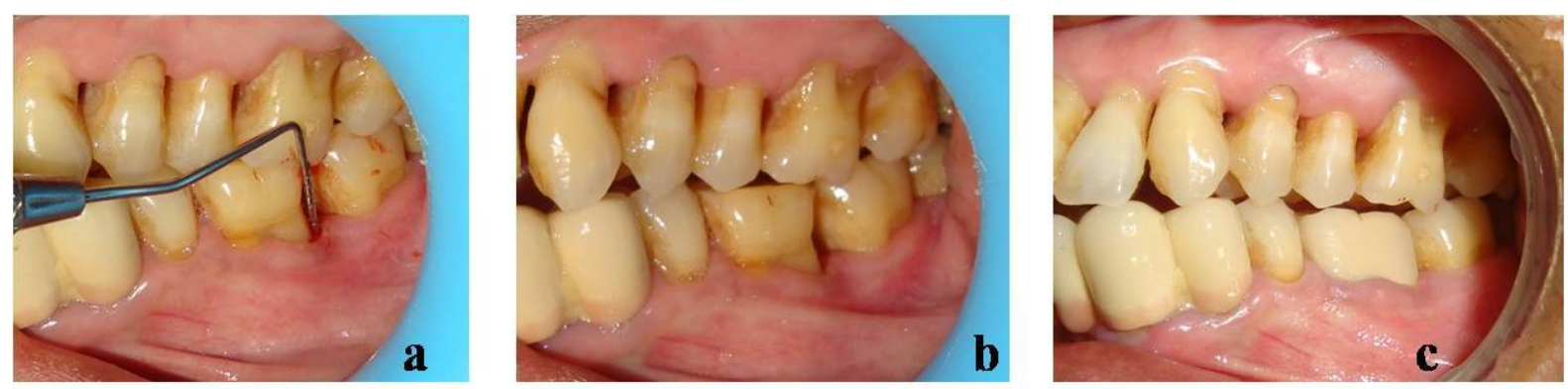

Figure 3. a- months after periodontal surgery, probing depth in 36 was reduced to 3mm; b- Prior to placement of PFM in 36; c- 24months following placement of PFM in 36

\section{Discussion}

Simring and Goldberg in 1964 [8] were the first to describe relationship between pulp and periodontium. The diagnostic challenge posed by endo-perio lesions could be overcome by proper history taking and sequential treatment planning. If the primary etiologic factor is endodontic, calcium hydroxide can be used as an intracanal medicament owing to its bactericidal, antiinflammatory and proteolytic property; it inhibits resorption; and it favours repair. It is especially effective in endodontic lesions with extensive periapical pathology and pseudo pockets, because of its temporary obturating action which would inhibit periodontal contamination of the instrumented canals via patent channels of communication. This regimen usually will resolve the pseudo pocket within a few weeks.

In this case the patient complained of food impaction in distal aspect of 36, there was no clinical or radiographic evidence of dental caries, isolated probing pocket depth of $10 \mathrm{~mm}$, radiographic evidence of bone loss beginning from the crest to apical region of 36 , pulp vitality test which showed the delayed response of the tooth to electric pulp testing indication partial vitality of the tooth helped in arriving at a provisional diagnosis of primary periodontal and secondary endodontic lesion [9].

Generally, in a case of primary periodontal and secondary endodontic lesion, an adequate endodontic therapy will aid in healing of the endodontic component, whereas and the prognosis of the tooth would ultimately depend on the efficacy of periodontal repair/regeneration initiated by either of the treatment procedures. In this case, following endodontic treatment the apical region in the IOPA showed signs of repair, but periodontal lesion (intra bony defect) and clinical parameters did not change after 3 months. The primary periodontal defect was later managed in a conservative approach with the use of regenerative techniques rather than the radical measures of hemisection of 36 .

One must distinguish an endodontic-periodontal combined lesion from a tooth with a vertical root fracture. A localized deep probing depth with minimal tooth mobility might have a favorable clinical outcome after the regenerative procedure. [10] Furthermore patient compliance in terms of maintaince of good oral hygiene also plays a pivotal role in the successful outcome of regenerative periodontal treatment.

\section{Conclusion}

This case report highlights that with the proper diagnosis and stringent case selection criteria, regenerative procedures could successfully treat an endodonticperiodontal combined lesion in a multi rooted mandibular molar. The treatment strategies and the clinical outcome depend on various factors including the extent of the periodontal disease, assessment of the therapeutic prognosis, with the intended regenerative procedure, tooth mobility, properly performed root canal treatment, and adequate healing time and patient compliance.

\section{References}

[1] Jansson L, Ehnevid H, Lindskog S, Blomlpf L. The influence of endodontic infection on progression of marginal bone loss in periodontitis. J Clin Periodontol 1995; 22: 729-73.

[2] Jansson L, Ehnevid H, Blomlpf L, Weintraub A, Lindskog S. Endodontic pathogens in periodontal disease augmentation. J Clin Periodontol 1995; 22: 598-602.

[3] Jansson L, Ehnevid H, Lindskog S, Bloml $\varphi$ f L. Relationship between periapical and periodontal status. A clinical retrospective study. J Clin Periodontol 1993; 20: 117-23.

[4] Simon JH, Glick DH, Frank AL. The relationship of endodonticperiodontic lesions. J Periodontol 1972; 43: 202-8.

[5] Richard E Walton and Mahmoud Torabinejad. Principles and Practice of Endodontics. 3rd Edition Philadelphia W B Saunders Company; 2002 pp. 467-84.

[6] Tonetti MS, Prato GP, Cortellini P. Factors affecting the healing response of intrabony defects following guided tissue regeneration and access flap surgery. J Clin Periodontol 1996; 23: 548-56.

[7] Trombelli L, Kim CK, Zimmerman GJ, Wikesjo UM. Retrospective analysis of factors related to clinical outcome of guided tissue regeneration procedures in intrabony defects. J Clin Periodontol 1997; 24: 366-71.

[8] Simring M, Goldberg M. The pulpal pocket approach: retrograde periodontitis. J Periodontol. 1964; 35: 22-48.

[9] Shenoy N, Shenoy A. Endo-perio lesions:Diagnosis and clinical considerations. Indian J Dent Res 2010; 21: 579-85.

[10] Se-Lim Oh, Ashraf F. Fouad, Sang-Hoon Park. Treatment Strategy for Guided Tissue Regeneration in Combined Endodontic-Periodontal Lesions: Case Report and Review. JOE 2009; 35(10): 1331-1336. 